\title{
Non-Gaussian Lineshapes and Dynamics of Time-Resolved Linear and Nonlinear (Correlation) Spectra
}

\author{
Mohammadhasan Dinpajooh $^{\dagger}$ and Dmitry V. Matyushov ${ }^{*, \dagger}$ \\ Department of Chemistry \& Biochemistry, Arizona State University, and Department of \\ Physics and Department of Chemistry 65 Biochemistry, Arizona State University, PO Box \\ 871504, Tempe, AZ 85287-1504 \\ E-mail: dmitrym@asu.edu
}

${ }^{*}$ To whom correspondence should be addressed

${ }^{\dagger}$ Department of Chemistry \& Biochemistry, Arizona State University

${ }^{\ddagger}$ Department of Physics and Department of Chemistry \& Biochemistry, Arizona State University, PO Box 871504, Tempe, AZ 85287-1504 


\begin{abstract}
Signatures of nonlinear and non-Gaussian dynamics in time-resolved linear and nonlinear (correlation) 2D spectra are analyzed in a model considering a linear plus quadratic dependence of the spectroscopic transition frequency on a Gaussian nuclear coordinate of the thermal bath (quadratic coupling). This new model is contrasted to the commonly assumed linear dependence of the transition frequency on the medium nuclear coordinates (linear coupling). The linear coupling model predicts equality between the Stokes shift and equilibrium correlation functions of the transition frequency and time-independent spectral width. Both predictions are often violated, and we are asking here the question of whether a nonlinear solvent response and/or non-Gaussian dynamics are required to explain these observations. We find that correlation functions of spectroscopic observables calculated in the quadratic coupling model depend on the chromophore's electronic state and the spectral width gains time dependence, all in violation of the predictions of the linear coupling models. Lineshape functions of 2D spectra are derived assuming Ornstein-Uhlenbeck dynamics of the bath nuclear modes. The model predicts asymmetry of $2 \mathrm{D}$ correlation plots and bending of the center line. The latter is often used to extract two-point correlation functions from 2D spectra. The dynamics of the transition frequency are non-Gaussian. However, the effect of non-Gaussian dynamics is limited to the third-order (skewness) time correlation function, without affecting the time correlation functions of higher order. The theory is tested against molecular dynamics simulations of a model polar-polarizable chromophore dissolved in a force field water.
\end{abstract}

Keywords: Nonlinear and non-Gaussian dynamics, time-resolved spectroscopy, 2D correlation spectroscopy, functional integrals, polarizable chromophores.

\section{Introduction}

Statistics and dynamics of the frequency of light absorption/emission in either visible/UV or infrared parts of the spectrum are widely used to study the nuclear dynamics of condensed media. Lineshapes of stationary optical absorption and emission report on the statistics of microscopic (molecularscale) fluctuations, while time-resolved changes of the lineshape (predominantly the line peak and width) report on their dynamics. ${ }^{1-4}$ In these types of experiments, linear time-resolved spectroscopies provide the time evolution of the spectral moments (one-point averages), ${ }^{4}$ while nonlinear spectroscopies give access to two-point time correlation functions of the transition frequency. ${ }^{5-8}$

Time evolution of the transition frequency directly reports on the dynamics of the thermal bath when it is a linear function of some subset of the bath nuclear coordinates (linear chromophore-bath coupling). Standard electrostatic models of solvation indeed suggest a linear coupling between permanent charges of the solute and the solvent. For instance, if the distribution of the chromophore's molecular charge is given by a dipole $\mathbf{m}_{0}$, it couples linearly to the electric field of the medium $\mathbf{E}$ and the electrostatic solute-solvent interaction en- ergy is simply $-\mathbf{m}_{0} \cdot \mathbf{E}{ }^{9,10}$ If the electric field is a Gaussian stochastic variable, i.e., only the first two cumulants of $\mathbf{E}$ contribute to the cumulant generating functional (line broadening function), $5,11,12$ the statistics of the transition frequency is Gaussian as well.

While the interaction of the solute dipole with the solvent electric field is linear, the free energy of polarizing the chromophore, $-\left(\alpha_{0} / 2\right) E^{2}$, scales quadratically with $E$ and linearly with the electronic polarizability of the chromophore $\alpha_{0} \cdot{ }^{10}$ When the dipolar and polarization terms are combined together, the solute-medium coupling is linear plus quadratic in the solvent field, which we call the "quadratic coupling" for brevity. As a consequence, the statistics of the energy gap ${ }^{13,14}$ and the time evolution of the spectral lineshape ${ }^{15,16}$ show non-Gaussian character even for a Gaussian thermal bath driving the transition. In other words, the statistics of the electric field $\mathbf{E}$ is Gaussian by virtue of long range electrostatic interactions involving many molecules (central limit theorem), but this Gaussian many-particle statistics is projected on non-Gaussian statistics of an internal variable (transition frequency) of a single/dilute solute when the coupling to the Gaussian thermal bath is nonlinear. 
The same quadratic dependence of the transition energy on nuclear coordinates appears when frequencies of a subset of nuclear modes change between two electronic states involved in the transition (Duschinsky's rotation ${ }^{17}$ ). Given that several physical mechanisms result in the same phenomenology, ${ }^{14}$ it is not surprising that nonGaussian statistics and/or nonlinear dynamics of spectral lineshapes, implying deviations from expectations of linear coupling models, have been recently reported for a number of systems. ${ }^{18-23}$

One faces, however, the dilemma of whether to assign the observations to either intrinsically nonGaussian fluctuations of the medium ${ }^{21,22,24}$ or to a nonlinear chromophore-medium coupling. ${ }^{13,14,25}$ However, the two perspectives can be merged into one question of what can be expected as observable consequences of non-Gaussian statistics and/or dynamics of the transition frequency, produced in either scenario, when recorded by linear and nonlinear spectroscopies. ${ }^{26}$

Addressing this question is the goal of this study. We report here on the development of a model based on the quadratic dependence of the transition frequency on a subset of Gaussian nuclear modes of the thermal bath. The model exactly sums up infinite series of cumulants and thus does not rely on truncated cumulant approximations. ${ }^{5}$ It can, therefore, attribute non-Gaussian lineshapes and their complex dynamics to either the non-Gaussian statistics of the transition frequency (originating from either the quadratic solute-solvent coupling or from changes of intrinsic solvent frequencies) or to non-Gaussian dynamics (existence of higherorder time correlation functions not reducible to the second-order one). Our agenda here is to provide a closed-form analytical framework for analyzing stationary and time-resolved, linear and nonlinear, spectroscopic lineshapes. The model's ability to incorporate both the non-Gaussian statistics and non-Gaussian dynamics significantly expands its reach compared to models based on the linear coupling to the thermal bath.

The quadratic solute-solvent coupling (known as the Q-model, "Q" for the quadratic term in the coupling) was previously applied to study the effects of non-Gaussian statistics of the donor-acceptor energy gap on electron-transfer reactions. ${ }^{14,27}$ The main property of interest in that problem is the equilibrium distribution $P(\Omega)$ of the transition frequency $\Omega$. The rate of an electron-transfer reaction is proportional to the probability $P(0)$ of radiation- less transition at $\Omega=0$. Two main distinctions from the traditional linear coupling models ${ }^{28}$ are seen as the asymmetry of the distribution and a linear exponential decay of the probability at the distribution's shallower wing, in contrast to the Gaussian quadratic exponential decay (Figure 1). Both non-Gaussian features arise from the summation of an infinite series of cumulants of $\Omega$, instead of applying a two-cumulant approximation. ${ }^{28}$ The question this model naturally poses is how this nonGaussian statistics extends to the realm of dynamics probed by linear time-resolved and non-linear correlation spectroscopies. ${ }^{5,7}$ This is the question addressed in this article.

Recent studies of vibrational lineshapes by $2 \mathrm{D}$ correlation spectroscopy ${ }^{7}$ have indicated that higher-order time correlation functions influence the time evolution of the observed lineshapes. ${ }^{21,24,29}$ There is also growing evidence that these non-Gaussian dynamics might be linked to quadratic solute-solvent coupling. The statistics of the vibrational frequency has been successfully mapped on the statistics of the medium electric field $E_{\|}$projected on the direction of vibrational stretch. ${ }^{19}$ This Stark-effect parameterization is usually achieved by fitting $\Omega\left(E_{\|}\right)$to a quadratic function of $E_{\| \cdot} \cdot{ }^{30,31}$ The electrostatic field is often found to be a nearly Gaussian stochastic variable, while the frequency becomes a non-Gaussian variable, both statistically and dynamically, because of the quadratic term in $\Omega\left(E_{\|}\right) .{ }^{29}$ The physical reason for the quadratic term in the Stark-effect parameterization is the polarizability of the vibrational mode. ${ }^{32}$ This is the same physics as was originally suggested in the Q-model of optical transitions ${ }^{13,15}$ and, given the Gaussian statistics of the fluctuating electric field, the mathematical formalism is identical in both applications.

Since the mathematical framework behind nonlinear/non-Gaussain spectral features reported by optical and vibrational spectroscopies might be common, we use here a physical system that is easier to implement in force field Molecular Dynamics (MD) simulations. Following our early studies of electron-transfer reactions, ${ }^{27}$ we consider a single solute carrying the dipole moment and polarizability. Both change with the electronic transition. By altering the relative magnitudes of changes in the dipole moment (linear coupling) and polarizability (quadratic coupling) one can adjust the statistics and dynamics of the transition energy from Gaussian (zero polarizability) to increasingly 
non-Gaussian. We stress, however, that the reach of the model is broader than this specific physical situation since it can be mapped on a number of phenomena involving the quadratic dependence of a collective coordinate on Gaussian nuclear modes of the thermal bath.

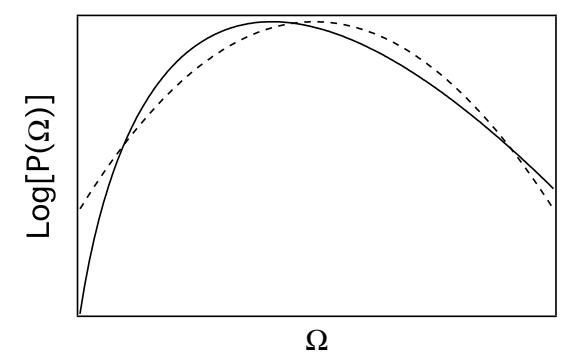

Figure 1: The distribution of transition frequencies in the Gaussian approximation (dashed line) and in the non-Gaussian Q-model ${ }^{14}$ (solid line). The Gaussian dashed line corresponds to the Q-model parameter $\alpha=\kappa / \Delta \kappa \rightarrow \infty$ (eqs (3) and (4)); the solid line was calculated with $\alpha=1.8$.

The standard formalism for setting up the dynamical equations of motion follows two steps. One first calculates the potential energy as a function of a dynamic coordinate. In the case of a collective coordinate (transition frequency $\Omega(t)$ in our case), this potential energy becomes the potential of mean force, a free energy. This part of the calculation is accomplished exactly within the Q-model. ${ }^{14}$ The potential of mean force $F(\Omega)=$ $-\beta^{-1} \ln [P(\Omega)]+$ Const is obtained from the statistical distribution function $P(\Omega)$, such as the one shown in Figure 1. The next step is to use $F(\Omega)$ to produce the mechanical force acting on the collective coordinate in an equation of motion describing its evolution. ${ }^{33}$ For the problem of fluctuating transition frequency, one can set up a Langevin equation for $\Omega(t)$ evolving in the potential $F(\Omega)$. The solution of the Langevin equation, or of the corresponding Fokker-Planck equation, ${ }^{11}$ would produce the propagator $P\left(\Omega, t \mid \Omega_{0}, 0\right)$ sufficient to calculate two-point time correlation functions of any order.

The second step in this program currently cannot be carried out exactly because of the lack of established solutions for stochastic dynamics in anharmonic potentials in general and in the one produced by the Q-model in particular (Figure 1). Therefore, for the sake of calculating the two-point correlation functions, we make an approximation following two steps. We first assume that the medium coordi- nate (electric field $\mathbf{E}$ or the coordinate $q$ below) is a Gaussian overdamped stochastic variable obeying the well-characterized Ornstein-Uhlenbeck stochastic process. ${ }^{11,34}$ We then project the known propagator $P\left(q, t \mid q_{0}, 0\right)$ on the coordinate $\Omega(q)$. While this approach allows an analytical solution for $2 \mathrm{D}$ correlation spectra, ${ }^{7}$ it is clearly an approximation when applied to time correlation functions, which needs testing against direct MD simulations. We will, therefore, start below with outlining the analytical formalism, followed by the simulation results.

\section{Time-resolved lineshapes}

We will consider a chromophore coupled to the nuclear mode $q$ of the medium and residing in either the ground (g) or excited (e) state. The Hamiltonian of the chromophore-medium system is $H_{g}$ in the ground state and $H_{e}$ in the excited state. The absorption of the radiation photon at $t=0$ results in the $\mathrm{g} \rightarrow \mathrm{e}$ transition with the time-dependent Hamiltonian

$$
H(t)=H_{g}+\hbar \Omega(q) \theta(t)
$$

Here, the vertical (Franck-Condon) transition frequency is

$$
\hbar \Omega(q)=H_{e}(q)-H_{g}(q)
$$

and $\theta(t)$ is the Heaviside step function.

We will further assume that each of the states is characterized by a Hamiltonian quadratic in the coordinate $q$, with both coefficients $C_{i}$ and $\kappa_{i}(i=$ g,e) changing with the excitation ${ }^{14}$

$$
H_{i}=I_{i}-C_{i} q+\left(\kappa_{i} / 2\right) q^{2}
$$

The transition frequency is then a quadratic function of $q$

$$
\hbar \Omega(q)=\hbar \Omega_{0}-\Delta C q+(\Delta \kappa / 2) q^{2}
$$

where $\hbar \Omega_{0}=I_{e}-I_{g}, \Delta C=C_{e}-C_{g}$, and $\Delta \kappa=$ $\kappa_{e}-\kappa_{g}$. The standard linear coupling models of spectroscopy assume $\Delta \kappa=0$. We will label this limit as the L-model ("L" for linear), while the case of $\Delta \kappa \neq 0$ will be labeled as the Q-model ("Q" for quadratic). ${ }^{14}$

Time-resolved linear spectroscopy monitors the 


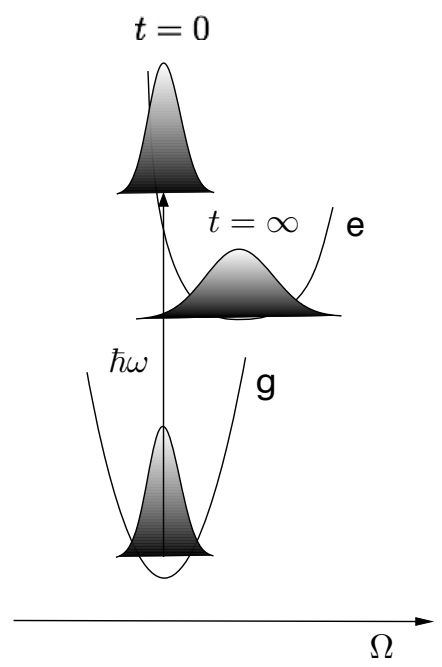

Figure 2: Time evolution of the lineshape after the ground state equilibrium distribution at $t=0$ is promoted, by photoexcitation, to a non-parabolic free energy excited surface. The time change of the spectral line-width is caused by a nonzero value of $\Delta \kappa$ in eq (4). The consequence of this term in the transition frequency is a non-Gaussian timedependent lineshape described by eq (24). An example calculation of the time-dependent line-width is shown in Figure 3.

time change of the spectral lineshape ${ }^{35}$

$$
I(\Omega, t)=\langle\delta[\Omega-\Omega(q(t))]\rangle_{t}
$$

where the average is taken over the evolving distribution function of the coordinate $q$ at time $t$.

Non-equilibrium distribution of nuclear coordinates is created by moving the ground state equilibrium distribution function

$$
P_{g}(\Omega)=\int \delta(\Omega-\Omega(q)) P_{g}(q) d q
$$

to the excited state potential curve at time $t=0$ (Figure 2); $P_{g}(q)$ is the equilibrium ground state distribution of $q$. The evolution of the nuclear coordinates of the system with the chromophore in its excited state is described by the conditional probability $^{11}$ (propagator) $P_{e}\left(q, t \mid q_{0}, 0\right)$. It gives the probability to find the nuclear coordinate with the value $q$ at $t=t$ given that it was $q_{0}$ at $t=0$. The average in eq (5) then becomes

$$
\langle\ldots\rangle_{t}=\int \ldots P_{e}\left(q, t \mid q_{0}, 0\right) P_{g}\left(q_{0}\right) d q d q_{0}
$$

Equation (7) projects the dynamics of the coordinate $q(t)$ on the dynamics of $\Omega(q(t)) .{ }^{15}$ There is no approximation involved in this procedure for calculating one-time averages, but it becomes approximate for two-time correlation functions as we discuss below.

There are a number of established results for Lmodels of spectroscopy based on the assumption of the Gaussian statistics of the stationary medium fluctuations and, for time-resolved measurements, of their Gaussian dynamics. ${ }^{22}$ The former assumes that only the first two cumulants of $q$ are significant for time-independent (stationary) lineshapes measured in the limit $t \rightarrow \infty$. The corresponding absorption and emission lineshapes are given by Gaussian functions, ${ }^{36}$ with their maxima separated by the Stokes shift $\Delta \Omega=\bar{\Omega}_{g}-\bar{\Omega}_{e}$,

$$
I_{i}(\Omega) \propto \exp \left[-\frac{\left(\Omega-\bar{\Omega}_{i}\right)^{2}}{2 \sigma^{2}}\right]
$$

Here, $\bar{\Omega}_{i}$ is the first spectral moment, equal to the position of the line maximum for Gaussian lineshapes. Further, the Gaussian width

$$
\sigma^{2}=\left\langle(\delta \Omega)^{2}\right\rangle=2 \lambda /\left(\beta \hbar^{2}\right)
$$

is related to the Stokes shift by the condition $2 \lambda=$ $\hbar \Delta \Omega ;{ }^{27} \lambda$ is the (nuclear) reorganization energy. ${ }^{37}$

Time-dependent lineshapes are often empirically approximated by Gaussian functions with a timedependent maximum $\bar{\Omega}_{i}(t)$ and a time-dependent spectral width $\sigma(t)^{16,38}$

$$
I_{i}(\Omega, t) \propto \exp \left[-\frac{\left(\Omega-\bar{\Omega}_{i}(t)\right)^{2}}{2 \sigma(t)^{2}}\right]
$$

The evolution of the maximum position gives the normalized dynamic Stokes-shift function

$$
S_{\Omega, i}(t)=\frac{\bar{\Omega}_{i}(t)-\bar{\Omega}_{i}(\infty)}{\bar{\Omega}_{i}(0)-\bar{\Omega}_{i}(\infty)}
$$

A similar function can be defined for the spectral width

$$
S_{\sigma}(t)=\frac{\sigma(t)^{2}-\sigma(\infty)^{2}}{\sigma(0)^{2}-\sigma(\infty)^{2}}
$$

where the stationary spectral width $\sigma(\infty)=\sigma$ (eq (9)) is reached in the limit $t \rightarrow \infty$.

The empirical Gaussian approximation of the time-dependent lineshape, as in eq (10), does not imply Gaussian dynamics of the transition frequency. The common meaning assigned to this term is the neglect of all time correlation functions $\left\langle\delta \Omega\left(t_{1}\right) \ldots \delta \Omega\left(t_{n}\right)\right\rangle$ with $n>2$ in the cumu- 
lant generating functional. Alternatively, this approximation implies expressing all even-order time correlation functions as powers of the two-time correlation function, known as Wick's theorem (odd correlation function vanish). ${ }^{39}$ What it practically means is known as the second-cumulant approximation, which replaces the generating functional of the transition frequency with the corresponding second cumulant ${ }^{5,12}$

$$
\begin{aligned}
e^{-g_{i}(t)}= & \left\langle\exp \left(-i \int_{0}^{t} d \tau \delta \Omega(\tau)\right)\right\rangle_{i} \\
& \simeq \exp \left[-\int_{0}^{t} d \tau \int_{0}^{\tau} d \tau^{\prime} C_{2, i}\left(\tau, \tau^{\prime}\right)\right]
\end{aligned}
$$

where $C_{2, i}\left(\tau, \tau^{\prime}\right)=\left\langle\delta \Omega(\tau) \delta \Omega\left(\tau^{\prime}\right)\right\rangle_{i}$. This approximation results in Kubo-type functions $g_{i}(t)$ evolving the spectral lineshape from a Lorentzian to a Gaussian on the relaxation time of the thermal bath. ${ }^{5,7}$ These models thus predict that $\sigma(t)$ reaches a constant value, $\sigma(t)=$ Const in eq (10), once the lineshape becomes Gaussian. There are, however, other approximations than just the second-cumulant approximation that are typically assumed in analyzing spectral dynamics.

It is commonly assumed that the correlation function $C_{2, i}(t)$ in eq (13) does not depend on the electronic state, ${ }^{40,41} C_{2, g}(t, 0)=C_{2, e}(t, 0)=C_{2}(t, 0)$, which is true for the L-models. Further, the linear response approximation ${ }^{42}$ in $\Omega(q)$ relates non-equilibrium dynamics of the spectral maximum to the equilibrium two-point correlation function, ${ }^{41,43,45}$ as is also derived in the Supporting Information (SI)

$$
S_{\Omega, i}(t)=S_{2}(t)
$$

where $S_{2}(t)=C_{2}(t, 0) / C_{2}(0,0)$. Equation (14) holds for either of the two states ${ }^{40,41,43}$ and, therefore, the index indicating the state has been dropped on its right-hand side. Since the secondcumulant approximation in eq (13) and the linear relation in eq (14) are not necessarily equivalent, we will reserve the term "Gaussian dynamics" for the former ${ }^{44}$ and "linear dynamics" for the latter. ${ }^{40,41,43,45}$

The linear response approximation also allows one to calculate $S_{\sigma}(t)$ in eq (12), which yields (see SI)

$$
S_{\sigma}(t)=\frac{(\beta \hbar \sigma)^{-1} S_{3}(t)+2 S_{2}(t)-S_{2}(t)^{2}}{1+(\beta \hbar \sigma)^{-1} S_{3}(0)}
$$

Here,

$$
S_{3}(t)=\sigma^{-3}\left\langle\delta \Omega(t)^{2} \delta \Omega(0)\right\rangle
$$

is the skewness correlation function, ${ }^{22}$ with the stationary spectral width $\sigma$ given by eq (9). Note that linear response does not stipulate Gaussian dynamics, and only the smallness of the perturbation introduced in the solvent by the electronic transition is required.

The linear function $\Omega(q)$ of the L-models directly relates the Stokes-shift correlation function in eq (11), calculated in the linear response approximation, to the medium dynamics

$$
S_{\Omega}(t)=S_{2}(t)=\chi(t)
$$

Here,

$$
\chi(t)=\sigma_{q}^{-2}\langle\delta q(t) \delta q(0)\rangle
$$

represents the dynamics of the bath and $\sigma_{q}^{2}=$ $\left\langle(\delta q)^{2}\right\rangle=(\beta \kappa)^{-1}$ is the variance of $q$. Equation (17) is, therefore, the basis for using spectroscopy to study the intrinsic dynamics of condensed media.

The results of applying the linear response approximation to the Stokes-shift dynamics are identical to the exact solution for the diffusive, overdamped dynamics over parabolic free energy surfaces obtained as a linear projection of $q(t)$ on the reaction coordinate $\Omega(t)$ (L-models). ${ }^{35,46}$ The dynamics of $q(t)$ is given by the stochastic OrnsteinUhlenbeck process with the propagator ${ }^{11,34}$

$$
P_{e}\left(q, t \mid q_{0}, 0\right) \propto \exp \left[-\frac{\beta \kappa_{e}}{2} \frac{\left(\delta q-\delta q_{0} \chi(t)\right)^{2}}{1-\chi(t)^{2}}\right]
$$

where $\delta q=q-q_{e}$ and $\delta q_{0}=q_{0}-q_{e}$ are the deviations of the, respectively, final and initial coordinates from the equilibrium value $q_{e}$ in the excited state. When this propagator is used in eqs (5) and (7) with $\kappa_{g}=\kappa_{e}$, one arrives at the linear response result for the Stokes-shift correlation function in eq (17) and, in addition, at a time-independent spectral width in eq (10)

$$
\sigma(t)=\text { Const }
$$

The physical meaning of eq (20) is straightforward: the relaxation of the ground state population, promoted to the exited surface with the same parabolic curvature as of the ground surface, produces no change in the distribution width (Figure 2 ). Only a time-dependent shift of the spectral maximum should be observed. Ornstein-Uhlenbeck dynamics of $\Omega(t)$ (L-models) also yield vanishing 
odd time correlation functions and a direct relation between higher order correlation functions and $S_{2}(t)$, for instance

$$
S_{4}(t)=\left\langle(\delta \Omega)^{4}\right\rangle^{-1}\left\langle\delta \Omega(t)^{2} \delta \Omega(0)^{2}\right\rangle=\frac{1}{3}+\frac{2}{3} S_{2}(t)^{2}
$$

This relation will be used below to test the Gaussian character of the dynamics of $\Omega(t)$ produced by MD simulations.

Equations (19) and (20) suggest that a nonlinear dependence $\Omega(q(t))$ on the Ornstein-Uhlenbeck stochastic variable $q(t)$ is required to produce a time-dependent width in the time-resolved lineshape in eq (10). This is indeed a feature of the dynamic version of the Q-model. ${ }^{15}$ Another consequence of this extension is the loss of a direct link between spectroscopic and bath dynamics, as we discuss below.

The quadratic solute-solvent coupling also makes the dynamics of the transition frequency nonGaussian. This can be demonstrated by calculating the skewness time correlation function (eq (16))

$$
S_{3, i}(t)=\left(\alpha_{i} \sqrt{2 \beta \lambda_{i}}\right)^{-1} \chi(t)(2+\chi(t))
$$

Here, $\alpha_{i}=\kappa_{i} / \Delta \kappa$ and

$$
\lambda_{i}=\beta \hbar^{2} C_{2, i}(0,0) / 2
$$

is the state-dependent reorganization energy. In deriving eq (22), the terms of the order $\left(\beta \lambda_{i}\right)^{-1}$ compared to the main contribution were dropped. When the same procedure is applied to $S_{4}(t)$, one arrives at the Gaussian formula for the correlation function in eq (21).

\section{Linear time-resolved spec- troscopy}

The time average in eq (5) can be directly carried out with the Ornstein-Uhlenbeck propagator in eq (19). The calculations are outlined in SI and here we only present the final result for the timedependent lineshape function ${ }^{15}$

$$
\begin{aligned}
I(\Omega, t) & \propto\left|\Omega-\omega_{0}\right|^{-1 / 2} e^{-\beta \hbar\left|\alpha(t)\left(\Omega-\omega_{0}\right)\right|} \\
& \times I_{1}\left(2 \beta \sqrt{\left|\alpha(t)^{3} \lambda(t) \hbar\left(\Omega-\omega_{0}\right)\right|}\right)
\end{aligned}
$$

Here, $I_{1}(x)$ is a modified Bessel function and the proportionality coefficient normalizes the lineshape. The $t \rightarrow \infty$ limit gives the stationary prob- ability of the transition frequency in the excited state $P_{e}(\Omega)$ shown by the solid line in Figure 1.

The lineshape function in eq (24) is clearly nonGaussian, with two time-dependent functions, $\lambda(t)$ and $\alpha(t)$. The former determine the width dynamics, $\sigma(t)^{2} \propto \lambda(t)$, the latter, $\alpha(t)=\kappa(t) / \Delta \kappa$, controls the extent of non-Gaussian character of the evolving lineshape. Here, $\kappa(t)$ is the dynamically evolving force constant of the medium coordinate $q$, which changes from $\kappa_{g}$ at $t=0$ to $\kappa_{e}$ at $t \rightarrow \infty$ (see SI). The parameter $\alpha(t)$ is inversely proportional to the change in the force constant $\Delta \kappa$ in eq (4). Therefore, eq (24) becomes a Gaussian function of eq (10) in the limit $\Delta \kappa \rightarrow 0$. In addition, the limiting frequency $\omega_{0}=\Omega_{0}-\Delta C^{2} /(2 \hbar \Delta \kappa)$ in eq (24), beyond which the Q-model intensity is identically zero, ${ }^{14}$ shifts to infinity at $\Delta \kappa \rightarrow 0$.

The reorganization function $\lambda(t)$ in eq (24) evolves in time between the initial, $\lambda_{g}$, and final, $\lambda_{e} \neq \lambda_{g}$, values given by eq (23) and, more specifically, by eq (S16) in SI. This time-dependent function (Figure 3 ) is given by the equation

$$
\lambda(t)=\frac{\kappa_{g}^{2}}{2 \kappa_{e}}\left(\frac{C_{e}}{\kappa_{e}}-\frac{C_{g}}{\kappa_{g}}\right)^{2} \zeta(t)\left[1+\frac{\Delta \kappa}{\kappa_{g}} \chi(t)^{2}\right]
$$

where

$$
\zeta(t)=\left[1+\left(\Delta \kappa / \kappa_{g}\right) \chi(t)\right]^{2}
$$

It is clear that $\lambda(t)=$ Const and $\sigma(t)=$ Const, in agreement with the result of L-models in eq (20), when $\kappa_{g}=\kappa_{e}$ and $\Delta \kappa=0$.

The non-Gaussian lineshape in eq (24) can often be represented by a time-dependent Gaussian function given by eq (10). The reorganization function $\lambda(t)$ then gives the time dependent linewidth $(\hbar \sigma(t))^{2}=2 \lambda(t) / \beta$, while the line maximum $\bar{\Omega}(t)$ is given by the relation

$$
\bar{\Omega}(t)=\omega_{0}+\zeta(t) \Delta C^{2} /(2 \hbar \Delta \kappa)
$$

From this equation, the Stokes-shift correlation function becomes

$$
S_{\Omega}(t)=\rho \chi(t)+(1-\rho) \chi(t)^{2}
$$

where $\rho=\left(2 \kappa_{g}\right) /\left(\kappa_{g}+\kappa_{e}\right)$. This function is a quadratic function in $\chi(t)$ and, obviously, is not the same as $\chi(t)$. Therefore, eq (17) does not hold and the Stokes-shift dynamics do not directly report on the medium dynamics. From eq $(25), S_{\sigma}(t)$ is a linear combination of powers of $\chi(t)$ up to the fourth order and one gets $S_{\sigma}(t) \neq S_{\Omega}(t)$. At the same 
time, $S_{\sigma}(t)$ becomes an algebraic function of $S_{\Omega}(t)$ and that relation can be used for testing the consistency of the observed dynamics with the predictions of the dynamic Q-model. Another important consequence of eq (28) is that the Stokes-shift dynamics are bi-exponential even if the medium dynamics are single-exponential.

Returning to equilibrium correlation functions, the Ornstein-Uhlenbeck propagator in eq (19) can be used to calculate $S_{2}(t)$ in the Q-model. The result, neglecting a small correction of the order $1 /\left(\beta \lambda_{i}\right)$, is $S_{2}(t)=\chi(t)$. We, therefore, obtain

$$
S_{\sigma}(t) \neq S_{\Omega}(t) \neq S_{2}(t)=\chi(t)
$$

We find the last equality to hold very accurately in our MD simulations of polarizable solutes in water presented below, even for a non-exponential $\chi(t)$.

We show in SI that the skewness correlation function does not contribute significantly to the linear response correlation function $S_{\sigma}(t)$ in eq (15), which then becomes a quadratic function of $\chi(t)$. On the other hand, $\sigma(t)^{2} \propto \lambda(t)$ in eq (25) is a fourth-order function in $\chi(t)$. Therefore, $S_{\sigma}(t)$ is of fourth order in $\chi(t)$ as well. We conclude that the linear response approximation cannot be applied to the width dynamics in the Q-model scenario.

It is useful to illustrate the analytical results with specific calculations. We show in Figure S3 in SI functions $S_{\Omega}(t)$ and $S_{\sigma}(t)$ plotted directly vs. $\chi(t)$ for different values of $\Delta \kappa / \kappa_{g}$. The deviations of two spectral functions from the bath dynamics increase with growing $|\Delta \kappa|$, but the effect of this quadratic coupling term on the width dynamics is more significant than on the Stokes-shift dynamics. This is also illustrated in Figure 3 in application to a more specific model related to our MD simulations.

Our simulations described below are done for a model solute in water, changing both its dipole moment and polarizability with photoexcitation. In anticipation of the specific results presented below, we use this model here to illustrate the nonlinear time evolution of the lineshape, which is not directly accessible by equilibrium MD simulations.

Figure 3 shows $\lambda(t)$, representing the evolution of the line width, and the Stokes-shift correlation function $S_{\Omega}(t)$ (inset in Figure 3). The parameters are chosen to allow the reorganization energy to increase by approximately a factor of two while evolving on the excited state surface (Figure 2). Despite this large increase in the spectral width, in contrast to expectations of the L-models (eq (20)), the

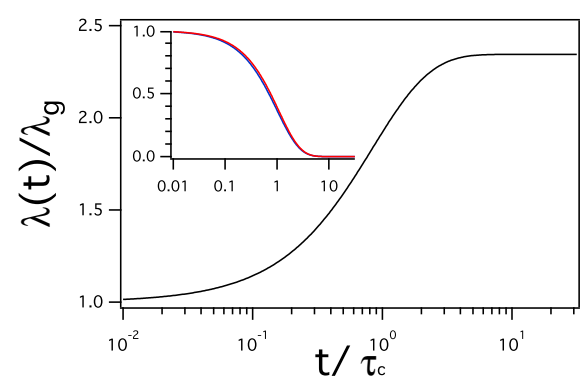

Figure 3: $\lambda(t)$ from eq (25). The inset shows $\chi(t)$ given by single exponential decay (eq (30), blue) and $S_{\Omega}(t)$ (eq $(28)$, red) vs. $t / \tau_{c}$. The calculations are done for a transition with $\Delta m / m_{g}=1$ and $\Delta \kappa / \kappa_{g}=-0.5 ; \lambda(0)=\lambda_{g}$ and $\lambda(\infty)=\lambda_{e}$.

Stokes-shift correlation function is nearly identical to $\chi(t)$ chosen in the form of a single-exponential decay

$$
\chi(t)=e^{-t / \tau_{c}}
$$

where $\tau_{c}$ is the relaxation time of the bath. ${ }^{1}$ Even though $S_{\Omega}(t)$ does not directly reproduce the medium dynamics (eq (29)), $S_{\Omega}(t)$ and $\chi(t)$ are very close, consistent with the MD results presented below.

To summarize, the Stokes-shift dynamics is a good reporter of the medium dynamics even for a quadratic solute-solvent coupling. Therefore, the time dependence of the spectral width should be used as an indicator of nonlinear dynamics. ${ }^{16}$ The next question is whether nonlinear correlation spectroscopy can provide a more sensitive tool. This question was partially addressed in the past, and it was shown ${ }^{21,24}$ that $2 \mathrm{D}$ spectra are not very sensitive to intrinsic non-Gaussian fluctuations of the thermal bath in L-models of spectroscopy. Below we explore a different scenario of Gaussian medium fluctuations combined with the Q-model of spectroscopy. Distinct and observable effects of nonlinear dynamics on 2D spectra are reported.

\section{D correlation spectroscopy}

\section{Line broadening function}

The lineshape of linear spectroscopy involves the average of the off-diagonal element of the density matrix $\rho_{g e}(t)$ over the individual molecules. This average defines the line broadening function $g(t)$ in eq (13). ${ }^{5,7}$ Third order response functions of nonlinear correlation spectroscopy involve terms, typ- 
ically represented by double sided Feynman diagrams, combining pure dephasing with population relaxation. We will consider only one such term here, since, for the two-state system, the rest of them can be obtained by changing the sign of the coherences in the dephasing diagrams. ${ }^{7}$ We, therefore, set up the calculation of the third-order lineshape function for the rephasing diagram (altering signs in the complex exponent)

$$
\begin{aligned}
\Psi\left(t_{1}, T, t_{2}\right)=\langle & \exp \left(-i \int_{0}^{t_{1}} d \tau \delta \Omega(\tau)\right) \\
& \left.\exp \left(i \int_{t_{1}+T}^{t_{1}+T+t_{2}} d \tau \delta \Omega(\tau)\right)\right\rangle
\end{aligned}
$$

where $t_{1}$ and $t_{2}$ are the durations of the pump and probe pulses, respectively, and $T$ is the population evolution, or waiting, time.

The frequency fluctuations are driven by the quadratic coupling to the stochastic variable $q$ performing overdamped fluctuations in a harmonic potential and thus described by the OrnsteinUhlenbeck process (eq (19)). ${ }^{11}$ Since the statistics and dynamics are non-Gaussian, the truncated cumulant approximation does not apply here and the problem needs to be directly integrated. This goal is achieved by path integration ${ }^{47}$ in the space of stochastic trajectories $q(\tau)$ as shown in SI. The path integral can be evaluated exactly for a quadratic $\Omega(q)$, leading to a novel analytical form for the line broadening function. We start the discussion with the standard L-model to set up the analytical framework for the next step incorporating the quadratic coupling with the bath.

For the L-model $(\Delta \kappa=0$ in eq (4)), eq (31) gives the Gaussian lineshape function ${ }^{1,5}$

$$
\Psi\left(t_{1}, T, t_{2}\right)=\exp \left[\phi\left(t_{1}, T, t_{2}\right)\right]
$$

Here,

$$
\phi\left(t_{1}, T, t_{2}\right)=-g\left(t_{1}\right)-g\left(t_{2}\right)^{*}+\chi(T) p\left(t_{1}\right) p\left(t_{2}\right)^{*}
$$

where asterisks denote complex conjugation. Further,

$$
g(t)=\left(\Delta \tau_{c}\right)^{2}\left[t / \tau_{c}-1+\chi(t)\right]
$$

with $(\hbar \Delta)^{2}=(\Delta C)^{2} \sigma_{q}^{2}$ is the standard Kubo's lineshape function ${ }^{1,5,7}$ and

$$
p(t)=\left(\Delta \tau_{c}\right)[1-\chi(t)]
$$

Equation (33), even though not presented in this form previously, is equivalent to the more commonly used relation ${ }^{5}$ obtained from the secondorder cumulant expansion of eq (31)

$$
\begin{aligned}
\phi\left(t_{1}, T, t_{2}\right) & =-g\left(t_{1}\right)-g\left(t_{2}\right)+g(T)-g\left(t_{1}+T\right) \\
& -g\left(t_{2}+T\right)+g\left(t_{1}+t_{2}+T\right)
\end{aligned}
$$

In the short-time approximation, neglecting the decay of the frequency correlations during the two coherence times $t_{1}$ and $t_{2}$, eq (33) reduces to the relation used in the past to model $2 \mathrm{D}$ lineshapes ${ }^{8,48-50}$

$$
\phi\left(t_{1}, T, t_{2}\right)=-\left(\Delta^{2} / 2\right)\left[t_{1}^{2}+t_{2}^{2}-2 \chi(T) t_{1} t_{2}\right]
$$

Further, eq (33) is derived for a rephasing response function in which the phase of the transition frequency switches from $i \delta \Omega(\tau)$ on the time interval $0 \leq \tau \leq t_{1}$ to complex conjugate $-i \delta \Omega(\tau)$ on the time interval $t_{1}+T \leq \tau \leq t_{1}+T+t_{2}$ (eq (31)). The non-rephasing diagrams preserve the same sign $-i \delta \Omega(\tau)$ on both time intervals. The non-rephasing $\tilde{\Psi}\left(t_{1}, T, t_{2}\right)$ will, therefore, be given by eq $(32)$ with the corresponding function $\tilde{\phi}\left(t_{1}, T, t_{2}\right)$ as follows

$$
\tilde{\phi}\left(t_{1}, T, t_{2}\right)=-g\left(t_{1}\right)-g\left(t_{2}\right)-\chi(T) p\left(t_{1}\right) p\left(t_{2}\right)
$$

The physical meaning of eq (33) is quite clear. Each function $g(t)$ describes the homogeneous and inhomogeneous broadening of lines produced by pump and probe pulses, while the last term shows the decay of coherence between them on the population relaxation time $T$, with the exponential time correlation function of the nuclear mode $\chi(T)=$ $\exp \left(-T / \tau_{c}\right)$. Given physical transparency of the equation, we will preserve its general structure when extending the calculations from the L-model to the Q-model.

Adopting the full quadratic form of $\Omega(q)$ of eq (4) still allows an exact analytical solution for the line broadening function (see SI). The main consequence of this extension is an appearance of an effective complex relaxation time $\tau_{c} / \epsilon$, where

$$
\epsilon^{2}=1-2 i \tau_{c} \Delta \kappa /(\kappa \beta \hbar)
$$

We show in SI that the functions $g(t)$ and $p(t)$ in 
eq (33) are replaced with

$$
\begin{aligned}
g(t) & =\left(\Delta \tau_{c}\right)^{2}\left[\frac{t}{\tau_{c}}-\frac{2}{\epsilon} \tanh \frac{\tilde{t}}{2}\right. \\
& \left.+\left(\frac{\epsilon^{2}+1}{2 \epsilon}\right)^{2} \frac{(\tanh (\tilde{t} / 2))^{2}}{1+\epsilon \operatorname{coth} \tilde{t}}\right]+\frac{p(\tilde{t})^{2}}{2}
\end{aligned}
$$

$\tilde{t}=\epsilon t / \tau_{c}$ and

$$
p(\tilde{t})=\left(\Delta \tau_{c}\right) \frac{\epsilon^{2}+1}{2 \epsilon} \frac{\cosh \epsilon \tilde{t}+\epsilon \sinh \tilde{t}-1}{\sinh \tilde{t}+\epsilon \cosh \tilde{t}}
$$

These functions reduce to the previous expressions when $\Delta \kappa=0$ and $\epsilon=1$.

The short-time approximation for $g(t)$ and $p(t)$ results in

$$
\begin{aligned}
\phi\left(t_{1}, T, t_{2}\right)= & -\left(\Delta^{2} / 8\right)\left[\left(c t_{1}\right)^{2}+\left(c^{*} t_{2}\right)^{2}\right. \\
& \left.-2|c|^{2} \chi(T) t_{1} t_{2}\right]
\end{aligned}
$$

where $c=1+\epsilon^{2}$. This equation becomes eq (37) of L-models at $\Delta \kappa=0$ and $\epsilon=1$. The magnitude of $\epsilon$ can, however, be fairly large at the typical conditions of optical experiment. Given that $\beta \hbar \simeq 2.5 \times 10^{-14} \mathrm{~s}$ and $\Delta \kappa / \kappa \simeq 1$, one expects $\epsilon^{2} \simeq-80 i$ at $\tau_{c} \simeq 1$ ps. For these large magnitudes of $\epsilon$, the short-time approximation in eqs (40) and (41) $t \ll \tau_{c} /|\epsilon|$ is limited to time-scales of tens of femtoseconds. However, for the polarizability of the $\mathrm{OH}$ stretch vibration, ${ }^{31}$ our estimate presented below gives $\Delta \kappa / \kappa=\alpha_{g}^{-1} \simeq-0.04$ and thus $\epsilon^{2} \simeq 1+3 i$.

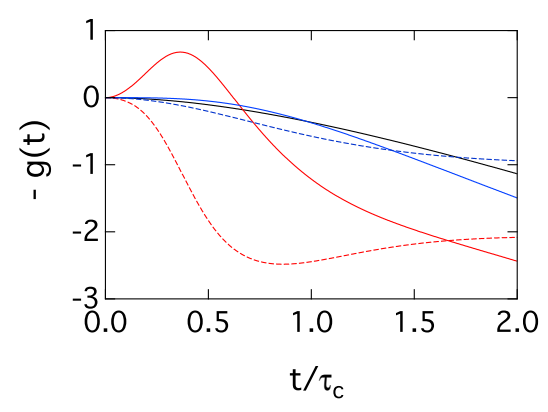

Figure 4: Function $-g(t) /\left(\Delta \tau_{c}\right)^{2}$ vs. $t / \tau_{c}$ for the Kubo lineshape (black, eq (34)) and for the Qmodel (eqs (40) and (41)). The parameters $\epsilon$ (eq (39)) is given as $\epsilon^{2}=1-i \epsilon^{\prime \prime}$ with $\epsilon^{\prime \prime}=2$ (blue) and $\epsilon^{\prime \prime}=10$ (red). The dashed lines refer to the imaginary part of $g(t)$.

That the short-time approximation becomes inaccurate for $|\epsilon| \gg 1$ is illustrated in Figure 4 where we compare the standard Kubo's line broadening function $-g(t)$ from eq (34) to $-g(t)$ from eq (40). The Q-model's $-g(t)$ develops a positive spike with increasing $|\epsilon|$, before turning into the negative territory, where it decays faster than Kubo's $-g(t)$. This shows that the quadratic approximation of eq (42) is insufficient since it does not give a convergent Fourier integral at imaginary and large in magnitude $\epsilon$; higher order expansion terms in $t$ are required. This comparison also implies that the approximate methods of extracting the medium correlation function $\chi(t)$ from either the eccentricity function or the slope of the center line obtained from correlation spectra, which are justified by the short-time approximation, ${ }^{8,49,50}$ are not applicable anymore as we discuss next.

\section{D lineshape}

We use here the broadening function derived above to produce $2 \mathrm{D}$ correlation spectra of a twostate system. ${ }^{7}$ The calculations are done for the commonly presented purely absorptive $2 \mathrm{D}$ spectrum given by the sum of the rephasing and non-rephasing spectra, after the inversion of the sign of the $\omega_{1}$ variable in the rephasing part, $R_{\text {abs }}\left(\omega_{1}, \omega_{3}\right) \propto \operatorname{Re}\left[R\left(-\omega_{1}, \omega_{3}, T\right)+\tilde{R}\left(\omega_{1}, \omega_{3}, T\right)\right]$. Each spectrum component here is obtained by Fourier transform of $\Psi\left(t_{1}, T, t_{2}\right)$ (eqs (32) and (33) for $R\left(-\omega_{1}, \omega_{3}, T\right)$ ) and $\tilde{\Psi}\left(t_{1}, T, t_{2}\right)$ (eq (38) for $\left.\tilde{R}\left(\omega_{1}, \omega_{3}, T\right)\right)$ in time variables $t_{1}$ and $t_{2}$.

Different metrics have been proposed to access the frequency time correlation function $S_{2}(t)$ directly from the evolution of $2 \mathrm{D}$ spectra in order to avoid fitting the lineshape to a predefined broadening function. These metrics themselves are largely justified on the basis of the short-time approximation $^{8,49,50}$ (eq (37)) and can be considered only as guidelines, disregarding the motional narrowing, to distinguish between different relaxation patterns. In particular, the eccentricity analysis ${ }^{8,49}$ is based on the ratio of the line widths measured along the diagonal $\omega_{1}=\omega_{3}$ direction $\left(\sigma_{\|}\right)$and along the antidiagonal direction $\left(\sigma_{\perp}\right)$. The short-time approximation then predicts access to the frequency correlation function of the bath $\chi(T)$ from the combination of the diagonal and antidiagonal widths as a function of the waiting time, $S_{E}(T)=S_{2}(T)=$ $\chi(T)$, where

$$
S_{E}(T)=\frac{\sigma_{\|}(T)^{2}-\sigma_{\perp}(T)^{2}}{\sigma_{\|}(T)^{2}+\sigma_{\perp}(T)^{2}}
$$


Repeating the arguments of ref $^{49}$ it is easy to show that a double Fourier transformation of the Q-model short-time expansion in eq (42) (under the conditions of convergence) should also yield $\chi(T)=S_{E}(T)$. The short-time approximation becomes, however, limited to very short times once an imaginary part is included in $\epsilon$, as is seen from the tail of $S_{E}(T)$ in Figure 5 deviating from $\chi(T)$ at longer waiting times. The eccentricity function does not correctly reproduce the frequency correlation function $S_{2}(t)=\chi(t)$ in the Q-model (filled circles in Figure 5), while this metric is quite reliable in L-models (open diamonds in Figure 5).

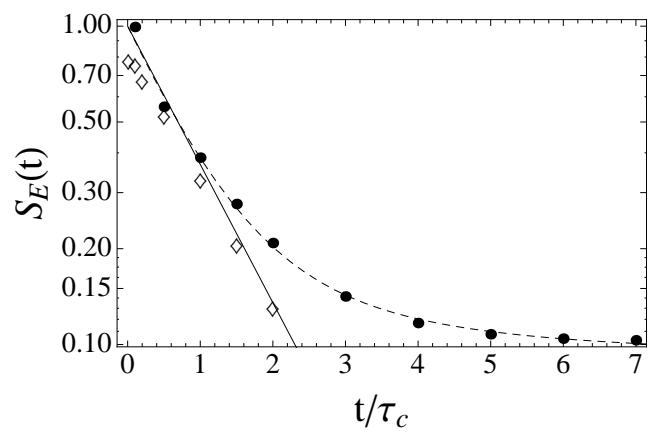

Figure 5: Time-dependent eccentricity function (eq (43)) calculated from the L-model (diamonds) and from the Q-model (circles). The solid line shows $S_{2}(t)=\chi(t)$ and the dashed line is a twoexponents plus a constant offset fit through the circles. The model parameters are the same as in Figure 4: $\Delta \tau_{c}=5, \epsilon^{2}=1+0.3 i$ for the Q-model and $\epsilon=1$ for the L-model.

An alternative approach to $S_{2}(t)$ is to measure the evolution of the slope of the center line. ${ }^{50,51}$ In this approach, cuts of $2 \mathrm{D}$ contours are made at constant $\omega_{1}$ and maxima of the profiles along the $\omega_{3}$ frequency are collected as a function of $\omega_{1}$. The time dependence of the slope of the center line gives access to $S_{2}(t) .{ }^{50}$ Recent experimental and theoretical studies, however, have shown that the center line develops a bend for some systems. ${ }^{22,23}$ A similar phenomenology follows from the dynamic Q-model developed here.

To demonstrate the new qualitative features introduced by the Q-model compared to the L-model, we use the parameters typical for vibrational spectroscopy and compare purely absorptive 2D spectra of a two-state system produced with the standard Kubo's broadening function (eqs (33)-(35)) to the same spectra obtained with the new broadening function derived here (eqs (33), (40), and (41)).
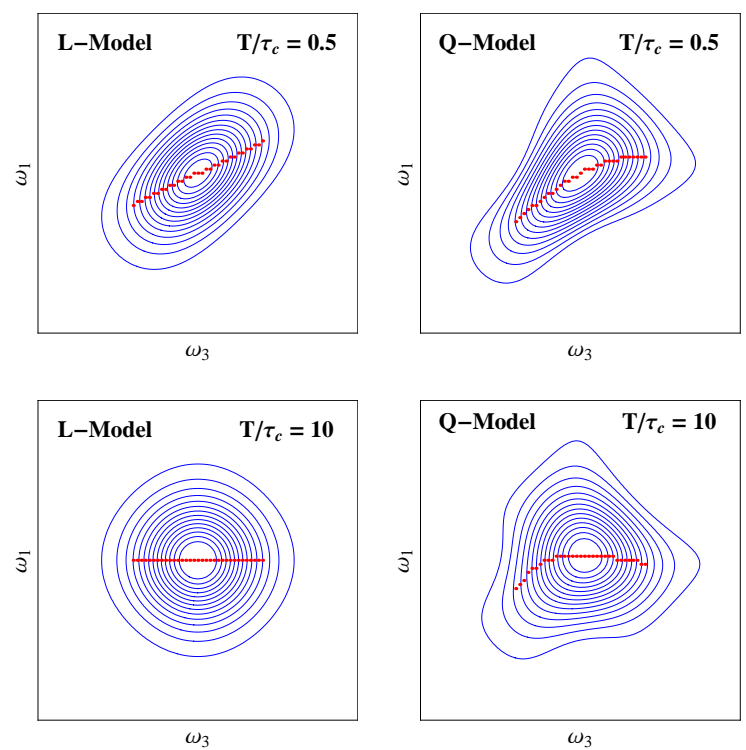

Figure 6: $2 \mathrm{D}$ spectra at different time delays $T$ calculated from L-model $(\epsilon=1)$ and Q-model $\left(\epsilon^{2}=\right.$ $1+0.3 i$ in eq (39)); $\Delta \tau_{c}=5$. The red dots indicate the center line.

These results are presented in four panels of Figure 6 , where the left two panels show the linear Kubo's result and the right two panels show the result of the Q-model (an additional Q-model correlation spectrum, with a higher $\epsilon$, can be found in SI). The main difference between the linear and quadratic models of line broadening is the asymmetry of the purely absorptive spectrum introduced by the complex $\epsilon$ in eq (39). The observable consequence is the bending of the center line.

\section{Numerical Simulations}

\section{Polar-polarizable chromophores}

In order to test the model by numerical simulations, a particular realization of the quadratic solute-solvent coupling due to solute's polarizability ${ }^{13,27}$ was used. The nuclear coordinate in this setup becomes the instantaneous electric field $\mathbf{E}$ of the solvent interacting with the solute dipole $\mathbf{m}_{0 i}$ and polarizing the solute as determined by its electronic polarizability $\alpha_{0 i}$ (assumed to be isotropic). Since both the dipole moment and the polarizability change with the electronic transition, the instantaneous transition frequency in eq (4) becomes

$$
\hbar \Omega(\mathbf{E})=\hbar \Omega_{0}-\Delta \mathbf{m}_{0} \cdot \mathbf{E}-\left(\Delta \alpha_{0} / 2\right) E^{2}
$$

where $\Delta \mathbf{m}_{0}=\mathbf{m}_{0 e}-\mathbf{m}_{0 g}$ and $\Delta \alpha_{0}=\alpha_{0 e}-\alpha_{0 g}$. 
The problem of electronic transitions in polarizable chromophores can be completely mapped on the Q-model if one additionally assumes that the distribution of the electric field is Gaussian. This implies that the term in the Hamiltonian describing fluctuations of the electric field inside a solute carrying no charges and polarizability is quadratic, $\left(4 a_{p}\right)^{-1} E^{2}$, where $a_{p}$ denotes the response coefficient (susceptibility) such that the chemical potential of solvating the dipole $m_{0 i}$ is $\mu_{i}=-a_{p} f_{i}\left(m_{0 i}\right)^{2}$. Here,

$$
f_{i}=\left[1-2 a_{p} \alpha_{0 i}\right]^{-1}
$$

is a factor accounting for the enhancement of the solute dipole due to an effective, mean-field addition of the induced and permanent dipoles. ${ }^{52}$ Therefore, if $\alpha_{0 i}=0, a_{p}$ is the linear susceptibility of the polar liquid solvent to the solute permanent dipole. It is given by the Onsager equation ${ }^{52}$ when the solute is a sphere of radius $R$ in a continuum dielectric with the static dielectric constant $\epsilon_{s}$

$$
a_{p}=\frac{1}{R^{3}} \frac{\epsilon_{s}-1}{2 \epsilon_{s}+1}
$$

The simulations reported below are performed in a non-polarizable force field of water. Therefore, the discussion is limited to a non-polarizable solvent. An extension to a more general case of polarizable solvents can be found elsewhere. ${ }^{13,15}$

With these assumptions, the Hamiltonians of the ground and excited states of the solute become

$$
H_{i}=I_{i}-\mathbf{m}_{0 i} \cdot \mathbf{E}-\left(\alpha_{0 i} / 2\right) E^{2}+\left(4 a_{p}\right)^{-1} E^{2}
$$

The mapping of the Q-model on eq (47) is straightforward and achieved by equating $\kappa_{i}$ in eq (3) to $\left(2 a_{p}\right)^{-1}-\alpha_{0 i}$ and $\Delta \kappa$ to $-\Delta \alpha_{0}$. The reorganization energies in two electronic states become

$$
\lambda_{i}=a_{p} f_{i}\left(\Delta \mathbf{m}_{0}+2 a_{p} f_{i} \Delta \alpha_{0} \mathbf{m}_{0 i}\right)^{2}
$$

In addition, the average transition frequencies are

$$
\hbar \bar{\Omega}_{i}=\hbar \Omega_{0}-2 a_{p} f_{i}\left(\Delta \mathbf{m}_{0} \cdot \mathbf{m}_{0 i}+a_{p} f_{i} \Delta \alpha_{0} m_{0 i}^{2}\right)
$$

The same equation can be written in a more compact form as

$$
\hbar \bar{\Omega}_{i}=\hbar \omega_{0}+\alpha_{i} \lambda_{i}
$$

where $\omega_{0}=\Omega_{0}+\left(\Delta m_{0}\right)^{2} /\left(2 \hbar \Delta \alpha_{0}\right)$ and

$$
\alpha_{i}=-\left(2 a_{p} f_{i} \Delta \alpha_{0}\right)^{-1}
$$

Before proceeding to MD simulations, we provide estimates of the typical values of $\alpha_{i}$ that might be seen in optical and IR spectroscopies. For optical absorption, $\Delta \alpha_{0}>0$ and can be of the same order of magnitude as the polarizability of the ground state $\Delta \alpha_{0} \sim \alpha_{0 g} \cdot{ }^{13,53}$ Given that polarizabilities of many organic molecules can be estimated as $\alpha_{0 g} \simeq R^{3} / 3,{ }^{54}$ one gets for the parameter controlling non-Gaussian behavior $\alpha_{g} \simeq-2$ in eq (51) $(\alpha=1.8$ is used in Figure 1). For $\mathrm{OH}$ stretch, the quadratic Stark effect results in ${ }^{31} \Delta \alpha_{0} \simeq 0.12 \AA^{3}$. Assuming $\Delta \alpha_{0} \simeq \alpha_{0 g}$ and $R \simeq 1.4 \AA$, one gets $\alpha_{g} \simeq-23$. Further, a nonlinear dependence of the vibrational frequency on the electric field, consistent with altering polarizability, was observed for $\mathrm{X}-\mathrm{H}$ group vibrations. ${ }^{32}$ The numerical estimate of $\Delta \alpha_{0}$ from these measurements is still a subject of uncertainty and cannot be used here for mapping on the Q-model.

The numerical simulations of electronic transitions in polarizable chromophores presented below separately address the statistics and dynamics of the transition frequency. We first start with analyzing the free energy surfaces of the two electronic states as functions of the transition frequency $\Omega$. Those are given in the Q-model by eq (24), in which the two stationary states are obtained by assigning $\alpha(t)$ to its initial, $\alpha_{g}=\alpha(t=0)$, and final, $\alpha_{e}=\alpha(t=\infty)$, values given by eq (51). We then proceed to the next step of analyzing the dynamics of the transition frequency, focusing in particular on nonlinear vs. non-Gaussian dynamics caused by the quadratic solute-solvent coupling of a polarizable chromophore.

\section{Free energy surfaces}

The system that we have chosen to analyze is made of a spherical solute with the radius $R=3 \AA$ dissolved in TIP4P water (Figure 7). The solute interacts with the oxygens of water by the LennardJones (LJ) potential and, additionally, carries two opposite charges $q$ separated by the distance $R=$ 2d. Polarizability of the solute is modeled by a Drude particle placed at its center (Figure 7). ${ }^{55}$ Isotropic polarizability of the solute $\alpha_{0}=q_{\mathrm{D}}^{2} / k_{\mathrm{D}}$ is achieved in simulations by allowing isotropic motions of the Drude particle constrained only by the potential energy penalty of stretching the spring with the force constant $k_{\mathrm{D}}$. The magnitude of the Drude particle charge $q_{\mathrm{D}}$ was changed to produce different values of $\alpha_{0}$. NAMD ${ }^{56}$ was used to in- 


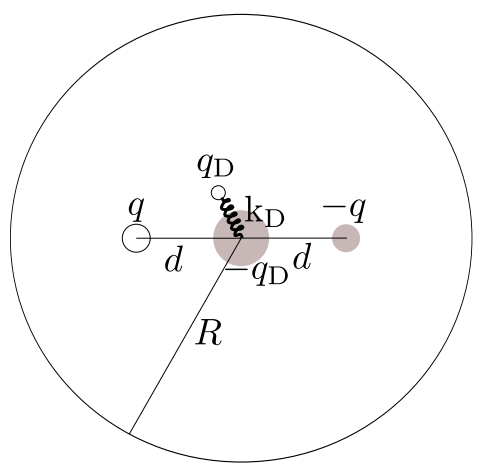

Figure 7: Polar-polarizable solute used in molecular dynamics simulations. A Lennard-Jones (LJ) solute of the radius $R$ contains two opposite charges separated by $2 d=R$. The negative charge $-q_{\mathrm{D}}$ at the center of the LJ solute is compensated by a Drude particle carrying the charge $+q_{\mathrm{D}}$. The charge and the spring constant $k_{\mathrm{D}}$ connecting the Drude particle to the LJ particle define the solute polarizability $\alpha_{0}=q_{\mathrm{D}}^{2} / k_{\mathrm{D}}$. Altering $q_{\mathrm{D}}$ is used to change the polarizability of the solute. The parameters of the solute and solvent force fields are given in the SI.

tegrate the MD trajectories. More details on the simulation protocol, force field parameters, and the analysis of the simulation trajectories are given in SI. Here, we proceed directly to the results.

Two sets of simulations were performed to test the model. In the first set, the dipole moment of the solute was varied at zero polarizability $\alpha_{0 i}=0$. The average solvent electric field as a function of $m_{0}$ then yields the susceptibility $a_{p}=0.0167 \AA^{-3}$ (see Figure S2 in SI). The Onsager equation (eq (46)) predicts $a_{p}=0.0181$ with $\epsilon_{s}=59$ of TIP4P water. ${ }^{57} \mathrm{~A}$ somewhat higher value from the Onsager equation with the dielectric cavity radius equated to the van der Waals radius of the solute is consistent with previous simulations. ${ }^{58}$ The simulations of polarizable chromophores were done in two dipolar states with $m_{0 g}=5 \mathrm{D}$ and $m_{0 e}=10$ $\mathrm{D}$ and the corresponding polarizabilities $\alpha_{0 g}=5$ $\AA^{3}$ and $\alpha_{0 e}=15 \AA^{3}$. For the sake of comparison, the same dipolar configurations of the solute were used to produce the free energy surfaces $F_{i}(\Omega)=-\beta^{-1} \ln \left[P_{i}(\Omega)\right]$ for polarizable and nonpolarizable chromophores (Figure 8 ).

The parabolic free energy surfaces of the Gaussian statistics (eq (8)) are calculated with $\alpha_{0 i}=0$ in eqs (48)-(50). The results are compared in Figure 8 a to direct sampling of the energy gap $\Omega(\mathbf{E})$ (eq (44)) along MD trajectories. Corresponding
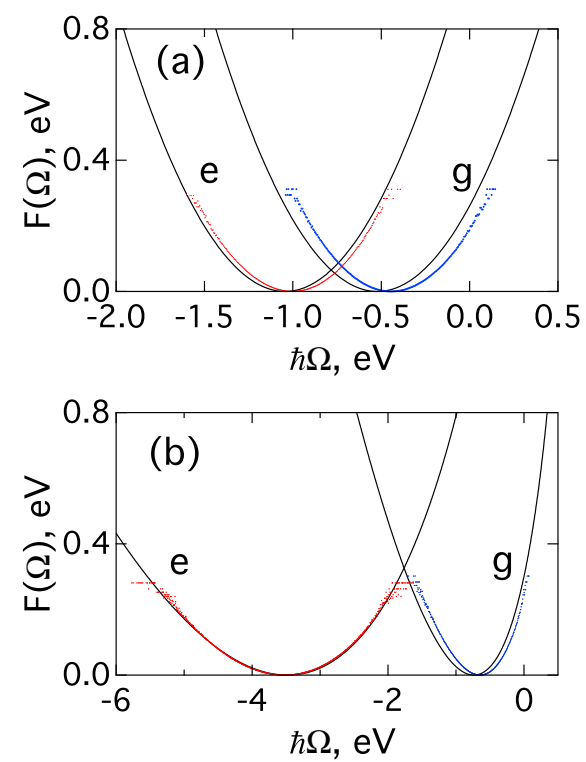

Figure 8: Free energy surfaces of the ground (g) and excited (e) states for non-polarizable (a) and polarizable (b) chromophores. The solid lines are the calculations with the Q-model using $a_{p}=$ $0.0167 \AA^{-3}$ as the sole input parameter. The dots represent distributions of the energy gap produced by MD simulations. All curves are brought to the same baseline at the positions of their minima.

free energy surfaces for polarizable chromophores are compared to eq (24) in Figure 8b. The results for the solvent-induced shifts $\Delta \Omega_{i}=\bar{\Omega}_{i}-\Omega_{0}$ and reorganization energies are listed in Table 1 . Simulations are in quantitative agreement with the Q-model for both purely dipolar and polarizable chromophores. A slight discrepancy between simulations and theory in the positions of the parabolas' minima at $m_{0 g}=5 \mathrm{D}$, seen in both cases, is probably caused by the non-point solute dipole in simulations and a corresponding contribution of the solute quadrupole to the solvation energy.

\section{Dynamics}

The free energy surfaces presented in Figure 8 suggest that dynamics of the transition frequency should slow down for states with higher solute polarizability. The curvature of $F_{i}(\Omega)$ is the restoring force constant of the harmonic motion, which loses its stiffness with increasing $\alpha_{0}$. The same statement applies to the dynamics of the solvent electric field E. The harmonic stiffness constant for the equations of field evolution is $\left(2 a_{p}\right)^{-1}-\alpha_{0 i}$ (eq (47)), and it decreases with increasing solute polar- 
Table 1: Solvent-induced spectral shifts and reorganization energies in two states of the polarizable and non-polarizable chromophore. The results of MD simulations are compared to calculations according to eqs (48)-(50) employing a single input parameter $a_{p}=0.0167 \AA^{-3}$ obtained from MD simulations of non-polarizable chromophores with varying magnitude of the dipole moment (Figure S2 in SI).

\begin{tabular}{lccccc}
\hline \multicolumn{2}{l}{ Parameters $^{a}$} & \multicolumn{2}{c}{ MD, eV } & \multicolumn{2}{c}{ Theory, eV } \\
$m_{0 i}$ & $\alpha_{0 i}$ & $-\hbar \Delta \Omega_{i}$ & $\lambda_{i}{ }^{b}$ & $-\hbar \Delta \Omega_{i}$ & $\lambda_{i}{ }^{b}$ \\
\hline 5 & 0 & 0.52 & 0.26 & 0.48 & 0.26 \\
10 & 0 & 1.04 & 0.26 & 1.04 & 0.26 \\
\hline $5(\mathrm{~g})^{c}$ & 5 & 0.68 & 0.57 & 0.75 & 0.62 \\
$10(\mathrm{e})^{c}$ & 15 & 3.54 & 2.83 & 3.51 & 2.88 \\
\hline
\end{tabular}

${ }^{a}$ Chromophore's dipole (D) and polarizability

$\left(\AA^{3}\right)$. ${ }^{b}$ Reorganization energies are calculated according to eq (9). ${ }^{c} \mathrm{~g}$ and e denote ground and excited states, respectively.

izability. As a result, the time correlation function $S_{\|}(t) \propto\left\langle\delta E_{\|}(t) E_{\|}(0)\right\rangle$ of the field projection on the solute dipole $E_{\|}(t)$ slows down with increasing solute polarizability (see Figure S5 in SI). ${ }^{59,60}$ This observation, goes beyond the mathematical framework of the dynamical Q-model following from the Ornstein-Uhlenbeck propagator in eq (19).

Equation (19) assumes that all the dependence of the dynamics of the transition frequency on the electronic state of the chromophore appears as a result of the quadratic coupling to the coordinate $q(t)$, the dynamics of which are not affected by changes in the chromophore. This assumption can of course be modified by assigning the dependence of the relaxation function $\chi(t)$ on the electronic state of the chromophore $\chi(t) \rightarrow \chi_{i}(t)$, $i=\mathrm{g}$, e. This alteration breaks the independence of the Stokes-shift and equilibrium correlation functions on the electronic state of the chromophore, but preserves the equality between $S_{2, i}(t)$ and $\chi_{i}(t)$. We find from our simulations that $S_{2, i}(t)$ matches $S_{\|, i}(t)$ really well. Points showing $S_{\|, i}(t)$ in Figure 9 essentially coincide on the plot scale with the solid lines representing $S_{2, i}(t)$. The origin of the dependence of $S_{2, i}(t)$ on the electronic state $i=\mathrm{g}$, e can, therefore, be assigned to the corresponding dependence of the dynamics of the nuclear coordinate.

The Q-model predicts distinctions between $S_{\sigma, i}(t), \quad S_{\Omega, i}(t)$, and $S_{2, i}(t)$ calculated in the same electronic state (eq (29)). All these predictions go beyond the standard expectations of L-models, ${ }^{40,41,43}$ implying that the dynamics are nonlinear. We find all these predictions to hold when tested against MD simulations: all correlation functions depend on the electronic state of the chromophore (cf. lines of different color in Figure 9) and the three correlation functions are different for the same electronic state (cf. different lines of the same color in Figure 9). However, $S_{\Omega, i}(t)$ is still a reasonable estimate of $\chi_{i}(t)$ and $S_{\sigma, i}(t)$ are close to both $S_{\Omega, i}(t)$ and $\chi_{i}(t)$. The main difference in the dynamics of two states comes from the dependence of $\chi_{i}(t)$ on the electronic state of the chromophore. The next question to address is whether higherorder time correlation functions are non-zero, i.e., whether the dynamics are non-Gaussian.

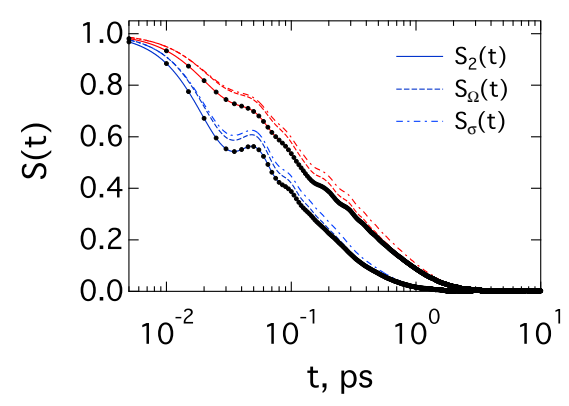

Figure 9: Normalized correlation functions calculated for the ground (blue, $m_{0 g}=5 \mathrm{D}$ and $\alpha_{0 g}=5$ $\AA^{3}$ ) and excited (red, $m_{0 e}=10 \mathrm{D}$ and $\alpha_{0 e}=15$ $\AA^{3}$ ) states of the polarizable chromophore. The solid lines refer to $S_{2, i}(t)$, with the superimposed dots showing the self-correlation function $S_{\|, i}(t)$ of the solvent field projected on the solute dipole. The dashed and dash-dotted lines show $S_{\Omega, i}(t)$ (eq $(28)$ ) and $S_{\sigma, i}(t)$ (eqs (12) and (25)), respectively.

We start by comparing the skewness time correlation function $S_{3, i}(t)$ (eq (16)), which is identically zero for Gaussian dynamics, between MD simulations and the Q-model (eq (22)). The Q-model pre$\operatorname{dicts} S_{3, i}(0) \propto-\Delta \alpha_{0}\left(\alpha_{i}^{-1} \propto \Delta \kappa, \Delta \kappa=-\Delta \alpha_{0}\right.$ in eq (22)). Since $\Delta \alpha=\alpha_{0 e}-\alpha_{0 g}>0$, it implies that $S_{3, i}(0)<0$. This is indeed observed in MD simulations for both $S_{3, g}(0)$ and $S_{3, e}(0)(\Omega(t)$ is defined by eq (44) for both states). Moreover, the agreement between the skewness functions calculated from MD and from the Q-model is nearly quantitative for the ground state (cf. dashed and solid blue lines in Figure 9). Further, eq (16) predicts that the skewness function should depend on the chromophore's state and, in particular, $S_{3, e}(0)$ 
should be smaller in the magnitude than $S_{3, g}(0)$ because of the larger reorganization energy of the exited state, $\lambda_{e}>\lambda_{g}$ (Table 1). This is indeed confirmed by $\mathrm{MD}$, but the alteration of $S_{3, e}(0)$ compared to $S_{3, g}(0)$ is greater in MD simulations than predicted by the Q-model. The agreement between the theory and simulations is only qualitative for the excited state skewness function.

The correlation functions $S_{4, i}(t)$ calculated from $\mathrm{MD}$ are practically indistinguishable from their corresponding Gaussian limits (eq (21), see Figure S6 in SI). This is the result of the fact that the nonGaussian corrections to eq (21) scale as $\left(\beta \lambda_{i}\right)^{-1}$ in $S_{4, i}(t)$ and the knowledge of the second-order correlation function $S_{2, i}(t)$ is sufficient to describe $S_{4, i}(t)$.

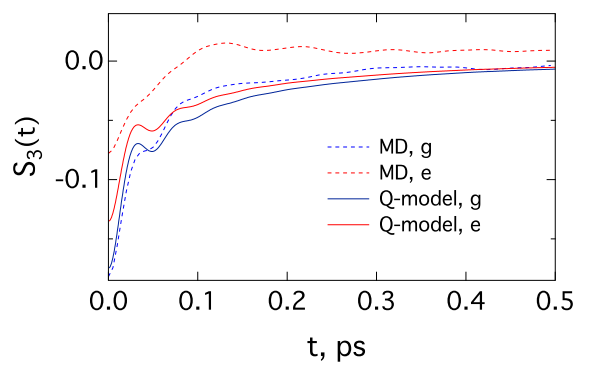

Figure 10: Skewness correlation function $S_{3, i}(t)$ (eq (16)) calculated from MD simulations (dashed lines) and from the Q-model (eq (22), solid lines). The calculations are done for the ground (blue, $m_{0 g}=5 \mathrm{D}$ and $\alpha_{0 g}=5 \AA^{3}$ ) and excited (red, $m_{0 e}=10 \mathrm{D}$ and $\alpha_{0 e}=15 \AA^{3}$ ) states of the polarizable chromophore. In both calculations, we have $\Delta m_{0}=5 \mathrm{D}$ and $\Delta \alpha_{0}=10 \AA^{3}$ in eq (44), consistent with the definition of the transition frequency in Figure 8. The time correlation function $\chi(t)=S_{\|}(t)$ from MD simulations of a nonpolarizable solute was used in eq (22) (see Figure S5 in SI).

The conclusion that can be drawn from calculations of time correlation functions is that the procedure adopted in the analytical model of projecting the Gaussian dynamics of nuclear medium coordinates on the transition frequency quadratic in these coordinates is generally supported by simulations. We also conclude that transient effects in timeresolved linear spectra going beyond the standard linear models, such as the dependence of the spectral width on time shown in Figure 3, arise from moving the equilibrium distribution belonging to one free energy surface to a free energy surface with a different curvature (Figure 2). The evolution of the excited-surface packet requires two correlation functions, $S_{2, e}(t)$ and $S_{3, e}(t)$. The effect of nonGaussian dynamics is, therefore, mostly limited to a non-zero skewness function $S_{3, i}(t)$ and does not affect the higher-order time correlation functions, which can be calculated based on the Gaussian dynamics.

\section{Discussion}

Two types of non-traditional dynamical effects of condensed materials on the time evolution of molecular spectra have recently come under scrutiny: (1) nonlinear dynamics and (2) non-Gaussian dynamics. The former puts under one umbrella all possible deviations from the results of L-models combined with the linear response approximation. The latter requires high-order time correlation functions not reducible to the second-order one.

Three consequences of nonlinear dynamics are typically recognized: (i) Stokes-shift correlation functions distinct from equilibrium correlation functions of both the transition frequency and the intrinsic nuclear coordinates of the thermal bath, ${ }^{41}$ (ii) differences between equilibrium time correlation functions in the ground and excited states of the chromophore, ${ }^{40,45}$ and (iii) time evolution of the spectral width. ${ }^{15,16,35}$ All these features are qualitatively reproduced by the Q-model.

The model achieves a more complete description of static and time-resolved lineshapes by summing an infinite series of spectral cumulants, instead of relying on the commonly applied two-cumulant approximation (zero cumulants beyond second order). The model yields non-Gaussian linear lineshapes (eq (24)) and predicts time evolution of the spectral linewidth (point (iii)).

The quadratic chromophore-medium coupling also requires a new analytical form for the line broadening function of $2 \mathrm{D}$ correlation spectra (eqs (33), (40), and (41)). Time evolution of 2D spectra shows bending of the center line and the deviation of the eccentricity function from the correlation function describing the bath dynamics. Given these complications, standard metrics of extracting the two-point correlation function from $2 \mathrm{D}$ spectra (center line, eccentricity function, etc.) do not apply here and direct fitting of $2 \mathrm{D}$ profiles to the line broadening function is required. Alternatives to direct fitting are clearly desirable, ${ }^{8,49-51}$ but have not been established so far for the Q-model. 
There is an important qualitative difference between two signatures of nonlinear dynamics: $S_{\Omega, i}(t) \neq S_{2, i}(t), S_{\sigma, i} \neq S_{\Omega, i}$ (point (i)) and $S_{2, g}(t) \neq S_{2, e}(t)$ (point (ii)). The former inequalities require going beyond the linear response (see SI), which is achieved here by summing an infinite series of transition frequency cumulants. By comparison, the difference between $S_{2, g}(t)$ and $S_{2, e}(t)$ can be accommodated within the linear response approximation once different force constants $\kappa_{i}$ for the nuclear coordinate are allowed in the ground and excited states (e.g., through solute's polarizability). The linear response approximation for these correlation functions requires the smallness of only the transition frequency $\Omega(t)$ relative to $H_{g}$ for absorption and relative to $H_{e}$ for emission (see SI). While linear expansion in $\Omega(t)$ is performed, different force constants $\kappa_{i}$ will project onto statedependent time correlation functions $\chi_{i}(t)$ of the nuclear mode. Even within the linear response approximation, different $S_{2, i}(t)$ will be produced for the ground and excited states.

The issue of non-Gaussian dynamics (point (2) above) is often entangled with nonlinear effects, but is in fact a separate issue. It is studied here by combining the dynamical Q-model with MD simulations. The third-order (skewness) time correlation function is non-zero for polarizable chromophores, thus corresponding to non-Gaussian dynamics of the transition frequency. The effect of non-Gaussian dynamics, within the present model, does not extend beyond the third-order correlation function.

Acknowledgement This research was supported by the National Science Foundation (CHE1213288). CPU time was provided by the National Science Foundation through XSEDE resources (TG-MCB080116N).

\section{Supporting Information Available:} Derivation of non-Gaussian lineshapes, simulation protocols, and data analysis. This material is available free of charge via the Internet at http://pubs.acs.org/.

\section{References}

(1) Kubo, R. In Adv. Chem. Phys.; Shuler, K., Ed.; Wiley Interscience, 1969; Vol. 15; p 101.
(2) Barbara, P. F.; Jarzeba, W. Ultrafast photochemical intramolecular charge and excited state solvation. Adv. Photochem. 1990, 15, 1.

(3) Bagchi, B.; Chandra, A. Collective orientational relaxation in dense dipolar liquids. $A d v$. Chem. Phys. 1991, 80, 1.

(4) Reynolds, L.; Gardecki, J. A.; Frankland, S. J. V.; Maroncelli, M. Dipole solvation in nondipolar solvents: Experimental studies of reorganization energies and solvation dynamics. J. Phys. Chem. 1996, 100, 10337-10354.

(5) Mukamel, S. Principles of Nonlinear Optical Spectroscopy; Oxford University Press: New York, 1995.

(6) Fleming, G. R.; Cho, M. Chromophoresolvent dynamics. Annu. Rev. Phys. Chem. 1996, 47, 109-134.

(7) Hamm, P.; Zanni, M. Concepts and Methods of $2 D$ Infrared Spectroscopy; Cambridge University Press: Cambridge, UK, 2011.

(8) Roberts, S. T.; Loparo, J. J.; Tokmakoff, A. Characterization of spectral diffusion from two-dimensional line shapes. J. Chem. Phys. 2006, 125, 084502-8.

(9) Mataga, N.; Kubota, T. Molecular interactions and electronic spectra; Marcel Dekker: New York, 1970.

(10) Liptay, W. In Modern quantum chemistry; Sinanoğlu, O., Ed.; Academic Press: New York, 1965.

(11) Gardiner, C. W. Handbook of stochastic methods; Springer: Berlin, 1997.

(12) Tanimura, Y. Stochastic Liouville, Langevin, Fokker-Planck, and Master Equation Approaches to Quantum Dissipative Systems. J. Phys. Soc. Jpn. 2006, 75, 082001.

(13) Matyushov, D. V.; Voth, G. A. A theory of electron transfer and steady-state optical spectra of chromophores with varying electronic polarizability. J. Phys. Chem. A 1999, 103, 10981.

(14) Matyushov, D. V.; Voth, G. A. Modeling the free energy surfaces of electron transfer in condensed phases. J. Chem. Phys. 2000, 113, 5413. 
(15) Matyushov, D. V. Time-resolved spectroscopy of polarizable chromophores. J. Chem. Phys. 2001, 115, 8933.

(16) Sajadi, M.; Obernhuber, T.; Kovalenko, S. A.; Mosquera, M.; Dick, B.; Ernsting, N. P. Dynamic Polar Solvation Is Reported by Fluorescing 4-Aminophthalimide Faithfully Despite H-Bonding. J. Phys. Chem. A 2009, 113, 44-55.

(17) Fischer, G. Vibronic coupling; Academic Press: London, 1984.

(18) Lawrence, C. P.; Skinner, J. L. Vibrational spectroscopy of $\mathrm{HOD}$ in liquid $\mathrm{D}_{2} \mathrm{O}$. II. Infrared line shapes and vibrational Stokes shift. J. Chem. Phys. 2002, 117, 8847.

(19) Fecko, C. J.; Eaves, J. D.; Loparo, J. J.; Tokmakoff, A.; Geissler, P. L. Ultrafast HydrogenBond Dynamics in the Infrared Spectroscopy of Water. Science 2003, 301, 1698-1702.

(20) Kwac, K.; Lee, H.; Cho, M. Non-Gaussian statistics of amide I mode frequency fluctuation of N-methylacetamide in methanol solution: Linear and nonlinear vibrational spectra. J. Chem. Phys 2004, 120, 1477.

(21) Bredenbeck, J.; Helbing, J.; Hamm, P. Solvation beyond the Linear Response Regime. Phys. Rev. Lett. 2005, 95, 083201.

(22) Roy, S.; Pshenichnikov, M. S.; Jansen, T. L. C. Analysis of 2D CS Spectra for Systems with Non-Gaussian Dynamics. J. Phys. Chem. B 2011, 115, 5431-5440.

(23) Dutta, S.; Li, Y.-L.; Rock, W.; Houtman, J. C. D.; Kohen, A.; Cheatum, C. M. 3-Picolyl Azide Adenine Dinucleotide as a Probe of Femtosecond to Picosecond Enzyme Dynamics. J. Phys. Chem. B 2012, 116, 542-548.

(24) Hamm, P. Three-dimensional-IR spectroscopy: Beyond the two-point frequency fluctuation correlation function. J. Chem. Phys. 2006, 124, 124506.

(25) Muljarov, E.; Zimmermann, R. Dephasing in Quantum Dots: Quadratic Coupling to Acoustic Phonons. Phys. Rev. Lett. 2004, 93, 237401.
(26) Garrett-Roe, S.; Hamm, P. What Can We Learn from Three-Dimensional Infrared Spectroscopy? Acc. Chem. Res. 2009, 42, 14121422.

(27) Small, D. W.; Matyushov, D. V.; Voth, G. A. The theory of electron transfer: What may be missing? J. Am. Chem. Soc. 2003, 125, $7470-7478$

(28) Marcus, R. A. On the theory of electrontransfer reactions. VI. Unified treatment for homogeneous and electrode reactions. $J$. Chem. Phys. 1965, 43, 679.

(29) Jansen, T. L. C.; Cringus, D.; Pshenichnikov, M. S. Dissimilar Dynamics of Coupled Water Vibrations. J. Phys. Chem. A 2009, 113, 6260-6265.

(30) Auer, B. M.; Skinner, J. L. IR and Raman spectra of liquid water: Theory and interpretation. J. Chem. Phys. 2008, 128, 224511-12.

(31) Yang, M.; Skinner, J. L. Signatures of coherent vibrational energy transfer in IR and Raman line shapes for liquid water. Phys. Chem. Chem. Phys. 2010, 12, 982-991.

(32) Saggu, M.; Levinson, N. M.; Boxer, S. G. Experimental Quantification of Electrostatics in $X-H \ldots \pi$ Hydrogen Bonds. J. Am. Chem. Soc. 2012, 134, 18986-18997.

(33) Chaikin, P. M.; Lubensky, T. C. Principles of condensed matter physics; Cambridge University Press: Cambridge, 1995.

(34) Wang, M. C.; Uhlenbeck, G. E. On the theory of the Brownian motion II. Rev. Mod. Phys. 1945, 17, 323-342.

(35) Stephens, M. D.; Saven, J. G.; Skinner, J. L. Molecular theory of electronic spectroscopy in nonpolar fluids: Ultrafast solvation dynamics and absorption and emission line shapes. $J$. Chem. Phys. 1997, 106, 2129-2144.

(36) Bixon, M.; Jortner, J. Electron transfer from isolated molecules to biomolecules. Adv. Chem. Phys. 1999, 106, 35.

(37) Marcus, R. A. Relation between charge transfer absorption and fluorescence spectra and the inverted region. J. Phys. Chem. 1989, 93, 3078-3086. 
(38) Richert, R. Triplet state solvation dynamics: Basics and applications. J. Chem. Phys. 2000, 113, 8404.

(39) Mahan, G. D. Many-Particle Physics; Plenum Press: New York, 1990.

(40) Carter, E. A.; Hynes, J. T. Solvation dynamics for an ion pair in a polar solvent: Time-dependent fluorescence and photochemical charge transfer. J. Chem. Phys. 1991, 94, 5961.

(41) Ladanyi, B. M.; Skaf, M. S. Computer simulation of hydrogen-bonding liquids. Annu. Rev. Phys. Chem. 1993, 44, 335.

(42) Hansen, J. P.; McDonald, I. R. Theory of Simple Liquids; Academic Press: Amsterdam, 2003.

(43) Laird, B. B.; Thompson, W. H. Timedependent fluorescence in nanoconfined solvents: Linear-response approximations and Gaussian statistics. J. Chem. Phys. 2011, 135, 084511.

(44) Ramasesha, K.; De Marco, L.; Horning, A. D.; Mandal, A.; Tokmakoff, A. A phenomenological approach to modeling chemical dynamics in nonlinear and two-dimensional spectroscopy. J. Chem. Phys. 2012, 136, 134507.

(45) Maroncelli, M.; Fleming, G. R. Computer simulation of the dynamics of aqueous solvation. J. Chem. Phys. 1988, 89, 5044-5069.

(46) Kinoshita, S. Theory of transient hole-burning spectrum for molecules in solution. J. Chem. Phys. 1989, 91, 5175-5184.

(47) Kleinert, H. Path Integrals in Quantum Mechanics, Statistics, Polymer Physics, and Financial Markets, 3rd ed.; World Scientific: New Jersey, 2004.

(48) Piryatinski, A.; Skinner, J. L. Determining Vibrational Solvation-Correlation Functions from Three-Pulse Infrared Photon Echoes. $J$. Phys. Chem. B 2002, 106, 8055-8063.

(49) Lazonder, K.; Pshenichnikov, M. S.; Wiersma, D. A. Easy interpretation of optical two-dimensional correlation spectra. Optics Letters 2006, 31, 3354-3356.
(50) Kwak, K.; Park, S.; Finkelstein, I. J.; Fayer, M. D. Frequency-frequency correlation functions and apodization in two-dimensional infrared vibrational echo spectroscopy: A new approach. J. Chem. Phys. 2007, 127, 124503.

(51) Kraemer, D.; Cowan, M. L.; Paarmann, A.; Huse, N.; Nibbering, E. T. J.; Elsaesser, T.; Miller, R. J. D. Temperature dependence of the two-dimensional infrared spectrum of liquid $\mathrm{H}_{2} \mathrm{O}$. Proc. Natl. Acad. Sci. USA 2008 , 105, 437-442.

(52) Onsager, L. Electric moments of molecules in liquids. J. Am. Chem. Soc. 1936, 58, 14861493.

(53) Renge, I. On the determination of molecular polarizability changes upon electronic excitation from the solvent shifts of absorption band maxima. Chem. Phys. 1992, 167, 173-184.

(54) Suppan, P. Excited-state dipole moments from absorption/fluorescence solvatochromic ratios. Chem. Phys. Lett. 1983, 94, 272-275.

(55) Lamoureux, G.; Roux, B. Modeling induced polarization with classical Drude oscillators: Theory and molecular dynamics simulation algorithm. J. Chem. Phys. 2003, 119, 30253039 .

(56) Phillips, J. C.; Braun, R.; Wang, W.; Gumbart, J.; Tajkhorshid, E.; Villa, E.; Chipot, C.; Skeel, R. D.; Kale, L.; Schulten, K. Scalable molecular dynamics with NAMD. J. Comp. Chem. 2005, 26, 17811802.

(57) Aragones, J. L.; MacDowell, L. G.; Vega, C. Dielectric Constant of Ices and Water: A Lesson about Water Interactions. J. Phys. Chem. A 2011, 115, 5745-5758.

(58) Matyushov, D. V. Dipole solvation in dielectrics. J. Chem. Phys. 2004, 120, 1375.

(59) Bursulaya, B. D.; Zichi, D. A.; Kim, H. J. Role of solute electronic polarizability in solvation dynamics. J. Phys. Chem. 1995, 99, 1006910074.

(60) Kumar, P. V.; Maroncelli, M. Polar solvation dynamics of polyatomic solutes: Simulation studies in acetonitrile and methanol. J. Chem. Phys. 1995, 103, 3038-3060. 